\title{
A semantical proof of the strong normalization theorem for full propositional classical natural deduction
}

\author{
Karim NOUR and Khelifa SABER \\ LAMA - Equipe de logique \\ Université de Chambéry \\ 73376 Le Bourget du Lac \\ e-mail : \{knour,ksabe $@ @$ univ-savoie.fr
}

\begin{abstract}
We give in this paper a short semantical proof of the strong normalization for full propositional classical natural deduction. This proof is an adaptation of reducibility candidates introduced by J.-Y. Girard and simplified to the classical case by M. Parigot.
\end{abstract}

\section{Introduction}

This paper gives a semantical proof of the strong normalization of the cut-elimination procedure for full propositional classical logic written in natural deduction style. By full we mean that all the logical connectives $(\perp, \rightarrow, \wedge$ and $\vee)$ are considered as primitive. We also consider the three reduction relations (logical, commutative and classical reductions) necessary to obtain the subformula property (see [5]).

Until very recently (see the introduction of [5] for a brief history), no proof of the strong normalization of the cut-elimination procedure was known for full logic.

In [5], Ph. De Groote gives such a proof by using a CPS-style transformation from full classical logic to implicative intuitionistic logic, i.e., the simply typed $\lambda$-calculus.

A very elegant and direct proof of the strong normalization of the full logic is given in [6] but only the intuitionistic case is given.

R. David and the first author give in 3 a direct and syntactical proof of this result. This proof is based on a characterization of the strongly normalizable deductions and a substitution lemma which stipulates the fact that the deduction obtained while replacing in a strongly normalizable deduction an hypothesis by another strongly normalizable deduction is also strongly normalizable. The same idea is used in 22 to give a short proof of the strong normalization of the simply typed $\lambda \mu$-calculus of $[9]$.

R. Matthes recently found another semantical proof of this result (see [7]). His proof uses a complicated concept of saturated subsets of terms.

Our proof is a generalization of M. Parigot's strong normalization result of the $\lambda \mu$-calculus (see 10]) for the types of J.-Y. Girard's system $\mathcal{F}$ using reducibility candidates. We also use a very technical lemma proved in $[3]$ concerning commutative reductions. To the best of our knowledge, this is the shortest proof of a such result.

The paper is organized as follows. In section 2, we give the syntax of the terms and the reduction rules. In section 3, we define the reducibility candidates and establish some important properties. In section 4, we show an "adequation lemma" which allows to prove the strong normalization of all typed terms. 


\section{The typed system}

We use notations inspired by the paper [1].

Definition 2.1 1. The types are built from propositional variables and the constant symbol $\perp$ with the connectors $\rightarrow, \wedge$ and $\vee$.

2. Let $\mathcal{X}$ and $\mathcal{A}$ be two disjoint alphabets for distinguishing the $\lambda$-variables and $\mu$-variables respectively. We code deductions by using a set of terms $\mathcal{T}$ which extends the $\lambda$-terms and is given by the following grammars:

$$
\begin{gathered}
\mathcal{T}:=\mathcal{X}|\lambda \mathcal{X} . \mathcal{T}|(\mathcal{T} \mathcal{E})|\langle\mathcal{T}, \mathcal{T}\rangle| \omega_{1} \mathcal{T}\left|\omega_{2} \mathcal{T}\right| \mu \mathcal{A} . \mathcal{T} \mid\left(\begin{array}{ll}
\mathcal{A} & \mathcal{T}
\end{array}\right) \\
\mathcal{E}:=\mathcal{T}\left|\pi_{1}\right| \pi_{2} \mid[\mathcal{X} . \mathcal{T}, \mathcal{X} . \mathcal{T}]
\end{gathered}
$$

An element of the set $\mathcal{E}$ is said to be an $\mathcal{E}$-term.

3. The meaning of the new constructors is given by the typing rules below where $\Gamma($ resp. $\Delta)$ is a context, i.e. a set of declarations of the form $x: A$ (resp. $a: A)$ where $x$ is a $\lambda$-variable (resp. a is a $\mu$-variable) and $A$ is a type.

$$
\begin{gathered}
\overline{\Gamma, x: A \vdash x: A ; \Delta}^{a x} \\
\frac{\Gamma, x: A \vdash t: B ; \Delta}{\Gamma \vdash \lambda x . t: A \rightarrow B ; \Delta} \rightarrow_{i} \quad \frac{\Gamma \vdash u: A \rightarrow B ; \Delta \quad \Gamma \vdash v: A ; \Delta}{\Gamma \vdash(u v): B ; \Delta} \rightarrow_{e} \\
\frac{\Gamma \vdash u: A ; \Delta}{\Gamma \vdash\langle u, v\rangle: A \wedge B ; \Delta} \wedge_{i} \\
\frac{\Gamma \vdash t: A \wedge B ; \Delta}{\Gamma \vdash\left(t \pi_{1}\right): A ; \Delta} \wedge_{e}^{1} \frac{\Gamma \vdash t: A \wedge B ; \Delta}{\Gamma \vdash\left(t \pi_{2}\right): B ; \Delta} \wedge_{e}^{2} \\
\frac{\Gamma \vdash t: A ; \Delta}{\Gamma \vdash \omega_{1} t: A \vee B ; \Delta} \vee_{i}^{1} \quad \frac{\Gamma \vdash t: B ; \Delta}{\Gamma \vdash \omega_{2} t: A \vee B ; \Delta} \vee_{i}^{2} \\
\frac{\Gamma \vdash t: A \vee B ; \Delta \quad \Gamma, x: A \vdash u: C ; \Delta \quad \Gamma, y: B \vdash v: C ; \Delta}{\Gamma \vdash(t[x . u, y . v]): C ; \Delta} \vee_{e} \\
\frac{\Gamma \vdash t: A ; \Delta, a: A}{\Gamma \vdash(a t): \perp ; \Delta, a: A} a b s_{i} \quad \frac{\Gamma \vdash t: \perp ; \Delta, a: A}{\Gamma \vdash \mu a . t: A ; \Delta} a b s_{e}
\end{gathered}
$$

4. The cut-elimination procedure corresponds to the reduction rules given bellow. There are three kinds of cuts:

(a) The logical cuts: They appear when the introduction of a connective is immediately followed by its elimination. The corresponding rules are:

- $(\lambda x . u \quad v) \triangleright u[x:=v]$

- $\left(\left\langle t_{1}, t_{2}\right\rangle \pi_{i}\right) \triangleright t_{i}$

- $\left(\omega_{i} t\left[x_{1} \cdot u_{1}, x_{2} \cdot u_{2}\right]\right) \triangleright u_{i}\left[x_{i}:=t\right]$

(b) The permutative cuts: They appear when the elimination of the disjunction is followed by the elimination rule of a connective. The corresponding rule is:

- $\left(\left(t\left[x_{1} \cdot u_{1}, x_{2} \cdot u_{2}\right]\right) \varepsilon\right) \triangleright\left(t\left[x_{1} \cdot\left(u_{1} \varepsilon\right), x_{2} \cdot\left(u_{2} \varepsilon\right)\right]\right)$ 
(c) The classical cuts: They appear when the classical rule is followed by the elimination rule of a connective. The corresponding rule is:

- $(\mu a . t \quad \varepsilon) \triangleright \mu a . t\left[a:={ }^{*} \varepsilon\right]$, where $t\left[a:={ }^{*} \varepsilon\right]$ is obtained from $t$ by replacing inductively each subterm in the form $(a v)$ by $(a(v \varepsilon))$.

Notation 2.1 Let $t$ and $t^{\prime}$ be $\mathcal{E}$-terms. The notation $t \triangleright t^{\prime}$ means that $t$ reduces to $t^{\prime}$ by using one step of the reduction rules given above. Similarly, $t \triangleright^{*} t^{\prime}$ means that $t$ reduces to $t^{\prime}$ by using some steps of the reduction rules given above.

The following result is straightforward.

Theorem 2.1 If $\Gamma \vdash t: A ; \Delta$ and $t \triangleright^{*} t^{\prime}$ then $\Gamma \vdash t^{\prime}: A ; \Delta$.

We have also the confluence property (see [1], 河 and [8]).

Theorem 2.2 If $t \triangleright^{*} t_{1}$ and $t \triangleright^{*} t_{2}$, then there exists $t_{3}$ such that $t_{1} \triangleright^{*} t_{3}$ and $t_{2} \triangleright^{*} t_{3}$.

Definition 2.2 An $\mathcal{E}$-term $t$ is said to be strongly normalizable if there is no infinite sequence $\left(t_{i}\right)_{i<\omega}$ of $\mathcal{E}$-terms such that $t_{0}=t$ and $t_{i} \triangleright t_{i+1}$ for all $i<\omega$.

The aim of this paper is to prove the following theorem.

Theorem 2.3 Every typed term is strongly normalizable.

In the rest of the paper we consider only typed terms.

\section{Reducibility candidates}

Lemma 3.1 Let $t, u$ and $u^{\prime}$ be $\mathcal{E}$-terms such that $u \triangleright u^{\prime}$, then:

1. $u[x:=t] \triangleright u^{\prime}[x:=t]$ and $u\left[a:={ }^{*} t\right] \triangleright u^{\prime}\left[a:={ }^{*} t\right]$.

2. $t[x:=u] \triangleright^{*} t\left[x:=u^{\prime}\right]$ and $t\left[a:={ }^{*} u\right] \triangleright^{*} t\left[a:={ }^{*} u^{\prime}\right]$.

Proof 1) By induction on $u$. 2) By induction on $t$.

Notation 3.1 The set of strongly normalizable terms (resp. $\mathcal{E}$-terms) is denoted by $\mathcal{N}$ (resp. $\left.\mathcal{N}^{\prime}\right)$. If $t \in \mathcal{N}^{\prime}$, we denoted by $\eta(t)$ the maximal length of the reduction sequences of $t$.We denote also $\mathcal{N}^{\prime<\omega}$ the set of finite sequences of $\mathcal{N}^{\prime}$.

Definition 3.1 Let $\bar{w}=w_{1} \ldots w_{n} \in \mathcal{N}^{\prime<\omega}$, we say that $\bar{w}$ is a nice sequence iff $w_{n}$ is the only $\mathcal{E}$-term in $\bar{w}$ which can be in the form $[x . u, y . v]$.

Remark 3.1 The intuition behind the notion of the nice sequences will be given in the proof of the lemma 3.3 .

Lemma 3.2 Let $\bar{w}=w_{1} \ldots w_{n}$ be a nice sequence and $\bar{w}^{\prime}=w_{1} \ldots w_{i}^{\prime} \ldots w_{n}$ where $w_{i} \triangleright w_{i}^{\prime}$. Then $\bar{w}^{\prime}$ is also a nice sequence.

Proof This comes from the fact that if $\varepsilon \triangleright[x . u, y . v]$ then $\varepsilon=[x . p, y . q]$, where $p \triangleright u$ or $q \triangleright v$. 
Notation 3.2 1. The empty sequence is denoted by $\emptyset$.

2. Let $\bar{w}=w_{1} \ldots w_{n}$ a sequence of $\mathcal{E}$-terms and $t$ a term. Then $(t \bar{w})$ is $t$ if $n=0$ and $\left(\left(t w_{1}\right) w_{2} \ldots w_{n}\right)$ if $n \neq 0$. The term $t\left[a:={ }^{*} \bar{w}\right]$ is obtained from $t$ by replacing inductively each subterm in the form $(a v)$ by $(a(v \bar{w}))$.

3. If $\bar{w}=w_{1} \ldots w_{n}$ is a nice sequence, we denote $\eta(\bar{w})=\sum_{i=1}^{n} \eta\left(w_{i}\right)$.

Lemma 3.3 Let $\bar{w}$ be a nice sequence.

1. $(x \bar{w}) \in \mathcal{N}$.

2. If $u \in \mathcal{N}$ and $(t[x:=u] \bar{w}) \in \mathcal{N}$, then $((\lambda x . t u) \bar{w}) \in \mathcal{N}$.

3. If $t_{1}, t_{2} \in \mathcal{N}$ and $\left(t_{i} \bar{w}\right) \in \mathcal{N}$, then $\left(\left(\left\langle t_{1}, t_{2}\right\rangle \pi_{i}\right) \bar{w}\right) \in \mathcal{N}$.

4. If $t, u_{1}, u_{2} \in \mathcal{N}$ and $u_{i}\left[x_{i}:=t\right] \in \mathcal{N}$, then $\left(\omega_{i} t\left[x_{1} . u_{1}, x_{2} . u_{2}\right]\right) \in \mathcal{N}$.

5. If $t\left[a:={ }^{*} \bar{w}\right] \in \mathcal{N}$, then $($ ra.t $\bar{w}) \in \mathcal{N}$.

\section{Proof}

1. Let $\bar{w}=w_{1} \ldots w_{n}$. All reduction over $(x \bar{w})$ take place in some $w_{i}$, because $\bar{w}$ is a nice sequence, and therefore the $w_{i}$ cannot interacte between them via commutative reductions. Since all $w_{i}$ are strongly normalizable, then $(x \bar{w})$ itself is strongly normalizable.

2. It suffices to prove that: If $((\lambda x . t u) \bar{w}) \triangleright s$, then $s \in \mathcal{N}$. We process by induction on $\eta(u)+\eta(t[x:=u] \bar{w})$. Since $\bar{w}=w_{1} \ldots w_{n}$ is a nice sequence, the $w_{i}$ cannot interact between them via commutative reductions. We have four possibilities for the term $s$.

- $s=\left(\left(\lambda x \cdot t^{\prime} u\right) \bar{w}\right)$ where $t \triangleright t^{\prime}$ : By lemma 3.1, $\left(t^{\prime}[x:=u] \bar{w}\right) \in \mathcal{N}$ and $\eta(u)+\eta\left(\left(t^{\prime}[x:=u] \bar{w}\right)\right)<\eta(u)+\eta((t[x:=u] \bar{w}))$, then, by induction hypothesis, $s \in \mathcal{N}$.

- $s=\left(\left(\lambda x . t u^{\prime}\right) \bar{w}\right)$ where $u \triangleright u^{\prime}$ : By lemma 3.1, $\left(t\left[x:=u^{\prime}\right] \bar{w}\right) \in \mathcal{N}$ and $\eta\left(u^{\prime}\right)+\eta\left(\left(t\left[x:=u^{\prime}\right] \bar{w}\right)\right)<\eta(u)+\eta((t[x:=u] \bar{w}))$, then, by induction hypothesis, $s \in \mathcal{N}$.

- $s=\left((\lambda x . t u) \bar{w}^{\prime}\right)$ where $\bar{w}^{\prime}=w_{1} \ldots w_{i}^{\prime} \ldots w_{n}$ and $w_{i} \triangleright w_{i}^{\prime}$ : By lemma 3.2, $\bar{w}^{\prime}$ is a nice sequence. We have $\left(t[x:=u] \bar{w}^{\prime}\right) \in \mathcal{N}$ and $\eta(u)+\eta((t[x:=$ $\left.\left.u] \bar{w}^{\prime}\right)\right)<\eta(u)+\eta((t[x:=u] \bar{w}))$, then, by induction hypothesis, $s \in \mathcal{N}$.

- $s=(t[x:=u] \bar{w})$ : By hypothesis, $s \in \mathcal{N}$.

3. Same proof as 2).

4. Same proof as 2).

5. It suffices also to prove that: If $(\mu a . t \bar{w}) \triangleright s$, then $s \in \mathcal{N}$. We process by induction on the pair $\left(\lg (\bar{w}), \eta\left(t\left[a:={ }^{*} \bar{w}\right]\right)+\eta(\bar{w})\right)$ where $\lg (\bar{w})$ is the number of the $\mathcal{E}$-terms in the sequence $\bar{w}$. We have three possibilities for the term $s$.

- $s=\left(\mu a . t^{\prime} \bar{w}\right)$ where $t \triangleright t^{\prime}:$ By lemma 3.1, $t^{\prime}\left[a:={ }^{*} \bar{w}\right] \in \mathcal{N}$ and $\eta\left(t^{\prime}\left[a:={ }^{*}\right.\right.$ $\bar{w}])<\eta\left(t\left[a:={ }^{*} \bar{w}\right]\right)$, then, by induction hypothesis, $s \in \mathcal{N}$.

- $s=\left(\mu a . t \bar{w}^{\prime}\right)$ where $\bar{w}^{\prime}=w_{1} \ldots w_{i}^{\prime} \ldots w_{n}$ and $w_{i} \triangleright w_{i}^{\prime}$ : by lemma 3.2, $\bar{w}^{\prime}$ is a nice sequence and, by lemma 3.1, $t\left[a:={ }^{*} \bar{w}^{\prime}\right] \in \mathcal{N}$ and $\eta(t[a:=*$ $\left.\left.\bar{w}^{\prime}\right]\right)+\eta\left(\bar{w}^{\prime}\right)<\eta\left(t\left[a:=^{*} \bar{w}\right]\right)+\eta(\bar{w})$, then, by induction hypothesis, $s \in \mathcal{N}$.

- $s=\left(\mu a . t\left[a:={ }^{*} w_{1}\right] \bar{w}^{\prime}\right)$ where $\bar{w}^{\prime}=w_{2} \ldots w_{n}$ : It is obvious that $\bar{w}^{\prime}$ is a nice sequence and $\lg \left(\bar{w}^{\prime}\right)<\lg (\bar{w})$. We have $t\left[a:={ }^{*} w_{1}\right]\left[a:={ }^{*} \bar{w}^{\prime}\right]=$ $t\left[a:=^{*} \bar{w}\right] \in \mathcal{N}$, then, by induction hypothesis, $s \in \mathcal{N}$. 
Lemma 3.4 Let $\bar{w}$ be a nice sequence.

$$
\text { If }(t[x .(u \bar{w}), y .(v \bar{w})]) \in \mathcal{N} \text {, then }((t[x . u, y . v]) \bar{w}) \in \mathcal{N} .
$$

Proof This is proved by that, from an infinite sequence of reduction starting from $((t[x . u, y . v]) \bar{w})$, an infinite sequence of reduction starting from $(t[x \cdot(u \bar{w}), y \cdot(v \bar{w})])$ can be constructed. A complete proof of this result is given in [3] in order to characterize the strongly normalizable terms.

Definition 3.2 1. We define three functional constructions $(\rightarrow, \wedge$ and $\vee$ ) on subsets of terms:

(a) $K \rightarrow L=\{t \in \mathcal{T} /$ for each $u \in K,(t u) \in L\}$.

(b) $K \wedge L=\left\{t \in \mathcal{T} /\left(t \pi_{1}\right) \in K\right.$ and $\left.\left(t \pi_{2}\right) \in L\right\}$.

(c) $K \vee L=\{t \in \mathcal{T} /$ for each $u, v \in \mathcal{N}:$ If (for each $r \in K, s \in L: u[x:=$ $r] \in \mathcal{N}$ and $v[y:=s] \in \mathcal{N})$, then $(t[x . u, y . v]) \in \mathcal{N}\}$.

2. The set $\mathcal{R}$ of the reductibility candidates is the smallest set of subsets of terms containing $\mathcal{N}$ and closed by the functional constructions $\rightarrow, \wedge$ and $\vee$.

3. Let $\bar{w}=w_{1} \ldots w_{n}$ be a sequence of $\mathcal{E}$-terms, we say that $\bar{w}$ is a good sequence iff for each $1 \leq i \leq n, w_{i}$ is not in the form $[x . u, y . v]$.

Lemma 3.5 If $R \in \mathcal{R}$, then:

1. $R \subseteq \mathcal{N}$.

2. $R$ contains the $\lambda$-variables.

Proof We prove, by simultaneous induction, that $R \subseteq \mathcal{N}$ and for each $\lambda$-variable $x$ and for each good sequence $\bar{w} \in \mathcal{N}^{\prime<\omega},\left(\begin{array}{ll}x & \bar{w}\end{array}\right) \in R$.

- $R=\mathcal{N}$ : trivial.

- $R=R_{1} \rightarrow R_{2}$ : Let $t \in R$. By induction hypothesis, we have $x \in R_{1}$, then $(t x) \in R_{2}$, therefore, by induction hypothesis, $(t x) \in \mathcal{N}$ hence $t \in \mathcal{N}$.

Let $\bar{w} \in \mathcal{N}^{\prime<\omega}$ be a good sequence and $v \in R_{1}$. Since $\bar{w} v$ is a good sequence, then, by induction hypothesis $(x \bar{w} v) \in R_{2}$, therefore $(x \bar{w}) \in R_{1} \rightarrow R_{2}$.

- $R=R_{1} \wedge R_{2}$ : Let $t \in R$, then $\left(t \pi_{i}\right) \in R_{i}$ and, by induction hypothesis, $\left(t \pi_{i}\right) \in \mathcal{N}$, therefore $t \in \mathcal{N}$.

Let $\bar{w} \in \mathcal{N}^{\prime<\omega}$ be a good sequence, then $\bar{w} \pi_{i}$ is also a good sequence and, by induction hypothesis, $\left(x \bar{w} \pi_{i}\right) \in R_{i}$, therefore $(x \bar{w}) \in R$.

- $R=R_{1} \vee R_{2}$ : Let $t \in R$ and $y, z$ two $\lambda$-variables. By induction hypothesis, we have, for each $u \in R_{1} \subseteq \mathcal{N}$ and $v \in R_{2} \subseteq \mathcal{N}, y[y:=u]=u \in \mathcal{N}$ and $z[z:=v]=v \in \mathcal{N}$, then $(t[y . y, z . z]) \in \mathcal{N}$, therefore $t \in \mathcal{N}$.

Let $\bar{w} \in \mathcal{N}^{\prime<\omega}$ be a good sequence and $u, v \in \mathcal{N}$ such that for each $r \in$ $R_{1}, s \in R_{2}, u[x:=r] \in \mathcal{N}$ and $v[y:=s] \in \mathcal{N}$. We have $[x . u, y . v] \in \mathcal{N}^{\prime}$ because $u$ and $v \in \mathcal{N}$. Thus $\bar{w}[x . u, y . v]$ is a nice sequence, and by lemma 3.3, $(x \bar{w}[x . u, y . v]) \in \mathcal{N}$, therefore $(x \bar{w}) \in R$. 
Notation 3.3 For $S \subseteq \mathcal{N}^{\prime<\omega}$, we define $S \rightarrow K=\{t \in \mathcal{T} /$ for each $\bar{w} \in S,(t \bar{w}) \in$ $K\}$.

Definition 3.3 A set $X \subseteq \mathcal{N}^{\prime<\omega}$ is said to be nice iff for each $\bar{w} \in X, \bar{w}$ is a nice sequence.

Lemma 3.6 Let $R \in \mathcal{R}$, then there exists a nice set $X$ such that $R=X \rightarrow \mathcal{N}$.

Proof By induction on $R$.

- $R=\mathcal{N}$ : Take $X=\{\emptyset\}$, it is clear that $\mathcal{N}=\{\emptyset\} \rightarrow \mathcal{N}$.

- $R=R_{1} \rightarrow R_{2}$ : We have $R_{2}=X_{2} \rightarrow \mathcal{N}$ for a nice set $X_{2}$. Take $X=\{u \bar{v} /$ $\left.u \in R_{1}, \bar{v} \in X_{2}\right\}$. We have $u \bar{v}$ is a nice sequence for all $u \in R_{1}$ and $\bar{v} \in X_{2}$. Then $X$ is a nice set and we can easly check that $R=X \rightarrow \mathcal{N}$.

- $R=R_{1} \wedge R_{2}$ : Similar to the previous case.

- $R=R_{1} \vee R_{2}$ : Take $X=\left\{[x . u, y . v] /\right.$ for each $r \in R_{1}$ and $s \in R_{2}, u[x:=$ $r] \in \mathcal{N}$ and $v[y:=s] \in \mathcal{N}\}$. We have $X$ is a nice set and, by definition, $R=X \rightarrow \mathcal{N}$.

Remark 3.2 Let $R \in \mathcal{R}$ and $X$ a nice set such that $R=X \rightarrow \mathcal{N}$. We can suppose that $\emptyset \in X$. Indeed, since $R \subseteq \mathcal{N}$, we have also $R=X \cup\{\emptyset\} \rightarrow \mathcal{N}$.

Definition 3.4 Let $R \in \mathcal{R}$, we define $R^{\perp}=\cup\{X / R=X \rightarrow \mathcal{N}$ and $X$ is a nice set $\}$.

Lemma 3.7 Let $R \in \mathcal{R}$, then:

1. $R^{\perp}$ is a nice set.

2. $R=R^{\perp} \rightarrow \mathcal{N}$.

\section{Proof}

1. By definition.

2. This comes also from the fact that: If, for every $i \in I, R=X_{i} \rightarrow \mathcal{N}$, then $R=\cup_{i \in I} X_{i} \rightarrow \mathcal{N}$.

Remark 3.3 For $R \in \mathcal{R}, R^{\perp}$ is simply the greatest nice $X$ such that $R=X \rightarrow \mathcal{N}$. In fact any nice $X$ such that $\emptyset \in X$ and $R=X \rightarrow \mathcal{N}$ would work as well as $R^{\perp}$.

Lemma 3.8 Let $R \in \mathcal{R}, t \in R$ and $t \triangleright^{*} t^{\prime}$. Then $t^{\prime} \in R$

Proof Let $\bar{u} \in R^{\perp}$. We have $(t \bar{u}) \triangleright^{*}\left(t^{\prime} \bar{u}\right)$ and $(t \bar{u}) \in \mathcal{N}$, then $\left(t^{\prime} \bar{u}\right) \in \mathcal{N}$. We deduce that $t^{\prime} \in R^{\perp} \rightarrow \mathcal{N}=R$. 
Remark 3.4 Let $R \in \mathcal{R}$, we have not in general $\mathcal{N} \subseteq R$, but we can prove, by induction, that $\mu a \mathcal{N}=\{\mu a . t / t \in \mathcal{N}$ and $a$ is not free in $t\} \subseteq R$.

\section{Proof of the theorem 2.3}

Definition 4.1 An interpretation is a function I from the propositional variables to $\mathcal{R}$, which we extend to any formula as follows: $I(\perp)=\mathcal{N}, I(A \rightarrow B)=I(A) \rightarrow$ $I(B), I(A \wedge B)=I(A) \wedge I(B)$ and $I(A \vee B)=I(A) \vee I(B)$.

Lemma 4.1 (Adequation lemma) Let $\Gamma=\left\{x_{i}: A_{i}\right\}_{1 \leq i \leq n}, \Delta=\left\{a_{j}: B_{j}\right\}_{1 \leq j \leq m}$, $I$ an interpretation, $u_{i} \in I\left(A_{i}\right), \bar{v}_{j} \in I\left(B_{j}\right)^{\perp}$ and $t$ such that $\Gamma \vdash t: A ; \Delta$.

Then $t\left[x_{1}:=u_{1}, \ldots, x_{n}:=u_{n}, a_{1}:={ }^{*} \overline{v_{1}}, \ldots, a_{m}:={ }^{*} v_{m}\right] \in I(A)$.

Proof For each term $s$, we denote

$s\left[x_{1}:=u_{1}, \ldots, x_{n}:=u_{n}, a_{1}:={ }^{*} \overline{v_{1}}, \ldots, a_{m}:={ }^{*} v_{m}^{-}\right]$by $s^{\prime}$.

We look at the last used rule in the derivation of $\Gamma \vdash t: A ; \Delta$.

- $\operatorname{ax}, \rightarrow_{e}$ and $\wedge_{e}^{j}$ : Easy.

- $\rightarrow_{i}$ : In this case $t=\lambda x$. $_{1}$ with $\Gamma, x: C \vdash t_{1}: D ; \Delta$ and $A=C \rightarrow D$. Let $u \in I(C)$ and $\bar{w} \in I(D)^{\perp}$. By induction hypothesis, we have $t_{1}^{\prime}[x:=$ $u] \in I(D)$, then $\left(t_{1}^{\prime}[x:=u] \bar{w}\right) \in \mathcal{N}$, and, by lemma $3.3\left(\left(\lambda x . t_{1}^{\prime} u\right) \bar{w}\right) \in \mathcal{N}$. Therefore $\left(\lambda x . t_{1}^{\prime} u\right) \in I(D)$, hence $\lambda x . t_{1}^{\prime} \in I(C) \rightarrow I(D)=I(A)$.

- $\wedge_{i}$ and $\vee_{i}^{j}$ : Similar to $\rightarrow_{i}$.

- $\vee_{e}:$ In this case $t=\left(t_{1}[x . u, y . v]\right)$ with $\Gamma \vdash t_{1}: B \vee C ; \Delta, \Gamma, x: B \vdash u: A ; \Delta$ and $\Gamma, y: C \vdash v: A ; \Delta$. Let $r \in I(B)$ and $s \in I(C)$. By induction hypothesis, we have $t_{1}^{\prime} \in I(B) \vee I(C), u^{\prime}[x:=r] \in I(A)$ and $v^{\prime}[y:=s] \in I(A)$. Let $\bar{w} \in I(A)^{\perp}$, then $\left(u^{\prime}[x:=r] \bar{w}\right) \in \mathcal{N}$ and $\left(v^{\prime}[y:=s] \bar{w}\right) \in \mathcal{N}$, therefore $\left(t_{1}^{\prime}\left[x .\left(u^{\prime} \bar{w}\right), y \cdot\left(v^{\prime} \bar{w}\right)\right]\right) \in \mathcal{N}$. By lemma 3.4. $\left(\left(t_{1}^{\prime}\left[x . u^{\prime}, y \cdot v^{\prime}\right]\right) \bar{w}\right) \in \mathcal{N}$, therefore $\left(t_{1}^{\prime}\left[x \cdot u^{\prime}, y \cdot v^{\prime}\right]\right) \in I(A)$.

- $a b s_{e}$ : In this case $t=\mu a . u$ and $\Gamma \vdash \mu a . u: A ; \Delta$. Let $\bar{v} \in I(A)^{\perp}$. It suffies to prove that $\left(\left(\mu a . u^{\prime}\right) \bar{v}\right) \in \mathcal{N}$. By induction hypothesis, $u^{\prime}\left[a:={ }^{*} \bar{v}\right] \in I(\perp)=\mathcal{N}$, then, by lemma 3.3, $\left(\mu a . u^{\prime} \bar{v}\right) \in \mathcal{N}$. Finally $(\mu a . u)^{\prime} \in I(A)$.

- $a b s_{i}$ : In this case $t=\left(a_{j} u\right)$ and $\Gamma \vdash\left(a_{j} u\right): \perp ; \Delta^{\prime}, a_{j}: B_{j}$. We have to prove that $t^{\prime} \in \mathcal{N}$, by induction hypothesis, $u^{\prime} \in I\left(B_{j}\right)$, then $\left(u^{\prime} \bar{v}_{j}\right) \in \mathcal{N}$, therefore $t^{\prime}=\left(a\left(u^{\prime} \bar{v}_{j}\right)\right) \in \mathcal{N}$.

Notation 4.1 We denote $I_{\mathcal{N}}$ the interpretation such that, for each propositional variable $X, I_{\mathcal{N}}(X)=\mathcal{N}$.

Proof [of theorem 2.3]: If $x_{1}: A_{1}, \ldots, x_{n}: A_{n} \vdash t: A ; a_{1}: B_{1}, \ldots, a_{m}: B_{m}$, then, by the lemma 3.5, $x_{i} \in I_{\mathcal{N}}\left(A_{i}\right)$, and, by definition, $\emptyset \in I_{\mathcal{N}}\left(B_{j}\right)^{\perp}$. Therefore by lemma 4.1, $t=t\left[x_{1}:=x_{1}, \ldots, x_{n}:=x_{n}, a_{1}:={ }^{*} \emptyset, \ldots, a_{m}:={ }^{*} \emptyset\right] \in I_{\mathcal{N}}(A)$ and finally, by lemma 3.5, $t \in \mathcal{N}$. 
Remark 4.1 We can give now another proof of remark 3.4: "if $R \in \mathcal{R}$, the

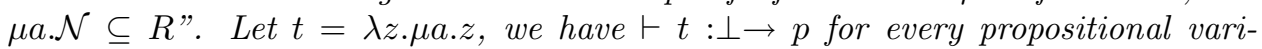
able p. By lemma 4.1, for every $R \in \mathcal{R}, t \in \mathcal{N} \rightarrow R$, then, for every $u \in \mathcal{N}$, $(t u) \in R$, therefore, by lemma 3.8, $\mu a . u \in R$.

\section{References}

[1] Y. Andou. Church-Rosser property of simple reduction for full first-order classical natural deduction. Annals of Pure and Applied logic 119 (2003) 225-237.

[2] R. David and K. Nour. A short proof of the strong normalization of the simply typed $\lambda \mu$-calculus. Schedae Informaticae vol.12, pp. 27-33, 2003.

[3] R. David and K. Nour. A short proof of the Strong Normalization of Classical Natural Deduction with Disjunction. Journal of symbolic Logic, vol 68, num 4, pp 1277-1288, 2003.

[4] J.-Y. Girard, Y. Lafont, P. Taylor. Proofs and types. Cambridge University Press, 1986.

[5] P. de Groote. Strong normalization of classical natural deduction with disjunction. In 5th International Conference on typed lambda calculi and applications, TLCA'01. LNCS (2044), pp. 182-196. Springer Verlag, 2001.

[6] F. Joachimski and R. Matthes. Short proofs of normalization for the simplytyped lambda-calculus, permutative conversions and Gödel's T. Archive for Mathematical Logic 42, pp 59-87 (2003).

[7] R. Matthes Non-strictly positive fixed-points for classical natural deduction. Manuscript, 2003.

[8] K. Nour and K. Saber Church-Russer property of full propositional classical natural deduction. Manuscript, 2004.

[9] M. Parigot $\lambda \mu$-calculus: An algorithm interpretation of classical natural deduction. Lecture Notes in Artificial Intelligence (624), pp. 190-201. Springer Verlag 1992.

[10] M. Parigot. Proofs of strong normalization for second order classical natural deduction. Journal of Symbolic Logic, 62 (4), pp. 1461-1479, 1997. 